\title{
Evaluation of mediastinal lymph nodes with endobronchial ultrasound: The thoracic surgeon's perspective
}

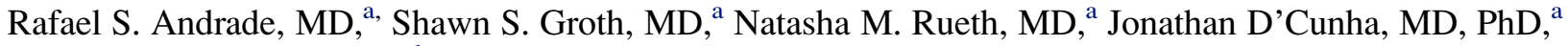 \\ Stefan E. Pambuccian, MD, ${ }^{\text {b }}$ and Michael A. Maddaus, MD, FACS ${ }^{\text {a }}$
}

\begin{abstract}
Objective: The objectives of our study are to (1) describe our experience with endobronchial ultrasound-guided fine-needle aspiration of mediastinal lymph nodes and (2) illustrate how thoracic surgeons facile with ultrasoundguided fine-needle aspiration have the potential to streamline patient care.
\end{abstract}

\begin{abstract}
Methods: We performed a retrospective review of all patients within our prospectively maintained database who underwent endobronchial ultrasound-guided fine-needle aspiration of mediastinal lymph nodes by thoracic surgeons at the University of Minnesota from September 1, 2006, to April 15, 2009. We included patients in our analysis if (1) their malignancy diagnosis was based on immediate endobronchial ultrasound-guided fine-needle aspiration cytology or (2) they underwent a confirmatory procedure (ie, mediastinoscopy or thoracoscopy) that sampled the same mediastinal lymph node stations biopsied by endobronchial ultrasound-guided fine-needle aspiration to verify normal, benign, or nondiagnostic endobronchial ultrasound-guided fine-needle aspiration findings. We also collected data on additional diagnostic or therapeutic procedures performed in the same anesthesia setting as endobronchial ultrasound-guided fine-needle aspiration.
\end{abstract}

Results: Over the study period, 192 patients underwent endobronchial ultrasound-guided fine-needle aspiration; 98 patients met our inclusion criteria. We achieved a sensitivity of $87.9 \%$, specificity of $97.4 \%$, and diagnostic accuracy of $91.7 \%$. For patients undergoing lung cancer staging, we sampled a mean of $3.0 \pm 0.9$ mediastinal lymph node stations. Half of our patients underwent an additional diagnostic or therapeutic procedure at the time of endobronchial ultrasound-guided fine-needle aspiration.

Conclusion: Thoracic surgeons who perform endobronchial ultrasound-guided fine-needle aspiration can achieve excellent sensitivity, specificity, and diagnostic accuracy while adhering to sound oncologic principles. Endobronchial ultrasound-guided fine-needle aspiration adds to the thoracic surgeon's unique capacity to expedite a diagnostic workup and treatment, thereby streamlining patient care. (J Thorac Cardiovasc Surg 2010;139: 578-83)

Real-time endobronchial ultrasound-guided fine-needle aspiration (EBUS-FNA) is a safe, simple, precise, minimally invasive method to diagnose mediastinal pathology and stage the mediastinum in thoracic malignancies. ${ }^{1}$ Although EBUS-FNA of mediastinal lymph nodes (MLNs) has a reported diagnostic performance that is comparable to mediastinoscopy, ${ }^{1-15}$ the available data have significant limitations. Concerns about EBUS-FNA of MLNs include the following: (1) The negative predictive value (NPV) for EBUS-FNA of MLNs may be poor (as low as $11 \%$ ) and may vary more widely than for mediastinoscopy; ${ }^{16}(2)$ published EBUS-FNA studies in patients undergoing mediasti-

From the Department of Surgery, ${ }^{\mathrm{a}}$ Division of General Thoracic and Foregut Surgery, and Department of Laboratory Medicine and Pathology, ${ }^{\mathrm{b}}$ University of Minnesota, Minneapolis, Minn.

Disclosures: None.

Received for publication May 2, 2009; revisions received Oct 29, 2009; accepted for publication Nov 13, 2009.

Address for reprints: Rafael S. Andrade, MD, Division of General Thoracic and Foregut Surgery, MMC 207, 420 Delaware St SE, Minneapolis, MN 55455 (E-mail: andr0119@umn.edu).

0022-5223/\$36.00

Copyright $\Subset 2010$ Published by Elsevier Inc. on behalf of The American Association for Thoracic Surgery

doi:10.1016/j.jtcvs.2009.11.017 nal staging for non-small cell lung cancer (NSCLC) report sampling on average only 1.5 central MLN stations per patient; ${ }^{7,17}$ and (3) to date, no reports have addressed performing additional diagnostic or therapeutic procedures in the same anesthesia setting as EBUS-FNA, in a manner akin to the current use of mediastinoscopy.

The aim of our study is to report our results with EBUSFNA of MLNs by addressing diagnostic performance and thorough staging. We also evaluated the use of additional procedures in the same anesthesia setting as EBUS-FNA in an attempt to demonstrate that thoracic surgeons facile in EBUS-FNA have the ability to streamline patient care.

\section{PATIENTS AND METHODS Patients}

The University of Minnesota Institutional Review Board approved this retrospective review of our prospectively maintained database and waived the need to obtain informed consent for each patient. We studied the charts of all consecutive patients who underwent EBUS-FNA at our institution for evaluation of mediastinal lymphadenopathy or staging of thoracic malignancy from September 1, 2006 (the first EBUS-FNA at the University of Minnesota), to April 15, 2009. We included patients in our analysis if (1) their malignancy diagnosis was based on immediate EBUS-transbronchial needle aspiration cytology or (2) they underwent a confirmatory procedure (ie, mediastinoscopy, thoracoscopy) that sampled the same MLN stations biopsied by EBUS-FNA to verify normal, benign, or nondiagnostic 


\section{Abbreviations and Acronyms \\ EBUS-FNA = endobronchial ultrasound-guided fine-needle aspiration \\ EUS = endoscopic ultrasound \\ FN $=$ false negative \\ FP $\quad=$ false positive \\ MLN = mediastinal lymph node \\ NPV $=$ negative predictive value \\ NSCLC = non-small cell lung cancer \\ ROSE $=$ rapid on-site evaluation \\ $\mathrm{TN}=$ true negative \\ $\mathrm{TP}=$ true positive}

EBUS-FNA findings (Figure 1). We excluded patients with benign or nondiagnostic biopsy results by EBUS-FNA who underwent a confirmatory procedure that sampled different MLN stations than those biopsied by EBUS-FNA, because we would not be able to verify our EBUS-FNA findings in these patients. We also excluded patients who underwent EBUSFNA of peribronchial masses. We collected the following information: (1) indications for EBUS-FNA; (2) immediate cytology (rapid on-site evaluation [ROSE]), permanent cytology, and histology findings; (3) number of MLN stations sampled, (4) short-axis diameter of sampled MLNs; (5) procedural time (defined as time elapsed between repositioning of the endotracheal tube by the surgeon at the beginning of the procedure and immediate cytopathologic diagnosis [ROSE]); and (6) additional confirmatory and therapeutic procedures performed in the same anesthesia setting.

\section{Endobronchial Ultrasound-Guided Fine-Needle Aspiration}

Our EBUS-FNA procedures were performed by 1 board-certified thoracic surgeon (R.S.A.) as previously described. ${ }^{2,15}$ Briefly, we perform the procedure under general anesthesia and sample each MLN station 3 times (10-15 passes per sample).

\section{Rapid On-Site Evaluation}

We rely on ROSE for intraoperative decision making because our 3 cytopathologists are experienced with transbronchial FNA specimens. As determined by the cytologist, the adequacy of cytology specimens is defined by the presence of lymphocytes; 40 lymphocytes per high-powered field or clusters of pigmented macrophages are strong predictors of an adequate cytology specimen. ${ }^{18}$ The absence of lymphocytes has a significant negative impact of the NPV of EBUS-FNA specimens slides (2 per sample), and all excess material is saved in formalin for further cytologic interpretation. ${ }^{19}$

\section{Statistical Analysis}

For overall diagnosis (based on ROSE), a true positive (TP) result was defined by the presence of any abnormal MLN pathology, whether benign (eg, granuloma or hyperplasia) or malignant. A true negative (TN) result was defined by the presence of a normal (and adequate) sample. If the sample was nondiagnostic or deemed normal after completion of EBUS-FNA but then abnormal after a confirmatory procedure, we defined the result as a false negative (FN). If the sample was deemed abnormal after completion of EBUSFNA but then normal after a confirmatory procedure, we defined the result as a false positive (FP). All calculations were made on a per patient basis.

For cancer diagnosis (based on ROSE), we defined a TP by the presence of malignant MLN pathology and a TN by a normal or benign (and adequate) sample and confirmation of nonmalignant diagnosis on surgical bi- opsy of the same MLN station. If the sample was nondiagnostic or deemed normal after completion of EBUS-FNA but then malignant after a confirmatory procedure, we defined the result as an FN. If the sample was deemed malignant after completion of EBUS-FNA but then normal after a confirmatory procedure, we defined the result as an FP.

For benign diagnosis (based on ROSE), we defined a TP by the presence of benign pathology (ie, granuloma, hyperplasia) and confirmation of that specific pathologic diagnosis with a surgical biopsy of the same MLN station. We defined a TN by a normal sample and confirmation of normal MLN histology on surgical biopsy of the same MLN station. If the sample was nondiagnostic or deemed normal after completion of EBUS-FNA but then pathologic (ie, granuloma, hyperplasia) after a confirmatory procedure, we defined the result as an FN. If the sample was deemed pathologic (ie, granuloma, hyperplasia) after completion of EBUS-FNA but then normal after a confirmatory procedure, we defined the result as an FP.

We used the standard definitions of sensitivity $(\mathrm{TP} /[\mathrm{TP}+\mathrm{FN}])$, specificity $(\mathrm{TN} /[\mathrm{TN}+\mathrm{FP}])$, positive predictive value $(\mathrm{PPV})(\mathrm{TP} /[\mathrm{TP}+\mathrm{FP}]), \mathrm{NPV}$ $(\mathrm{TN} /[\mathrm{TN}+\mathrm{FN}])$, and accuracy $([\mathrm{TP}+\mathrm{TN}] /[\mathrm{TP}+\mathrm{TN}+\mathrm{FP}+\mathrm{FN}])$.

To analyze our data, we used SAS version 9.1 (SAS Institute Inc, Cary, $\mathrm{NC}$ ). We report descriptive statistics as the median (range in parentheses) or mean \pm standard deviation.

\section{RESULTS \\ Patients}

During the study period, 192 patients underwent EBUSFNA, and 98 patients met our inclusion criteria. Indications for EBUS-FNA were mediastinal lymphadenopathy $(56.1 \%)$ and thoracic malignancy staging $(43.9 \%)$. On endoscopic evaluation, the median short-axis MLN diameter was $11 \mathrm{~mm}$ (range, $3-30 \mathrm{~mm}$ ). Table 1 lists the final pathologic diagnoses.

\section{Performance Measures}

We found a higher sensitivity, NPV, and accuracy for malignant MLN pathology than for benign pathology (Table 2). ROSE findings were identical to permanent cytology findings in 92 patients $(94.8 \%)$ (Table 3). Of the 33 patients with NSCLC, $14(14.4 \%)$ underwent EBUS-FNA to diagnose recurrence; 19 patients $(19.6 \%)$ underwent initial staging EBUS-FNA before any form of therapy or underwent restaging after neoadjuvant therapy. In these 19 patients, the mean number of central MLN stations sampled was $3.0 \pm 0.9$. One patient with NSCLC had an FN ROSE in an N1 (11R) lymph node.

\section{Efficiency of Patient Care}

The total procedure time (including pathologic diagnosis) was recorded in 31 patients (mean, $46.5 \pm 20.5$ minutes); the procedure time per MLN station was $20.8 \pm 10.5$ minutes. We performed 88 additional procedures in 49 patients $(50 \%)$ in the same anesthesia setting as EBUS-FNA (Table 4). Because a malignant diagnosis was made on ROSE, 53 patients $(54 \%)$ avoided a more invasive diagnostic procedure.

\section{DISCUSSION}

Our report demonstrates that thoracic surgeons can (1) accurately use EBUS-FNA, (2) follow appropriate oncologic 


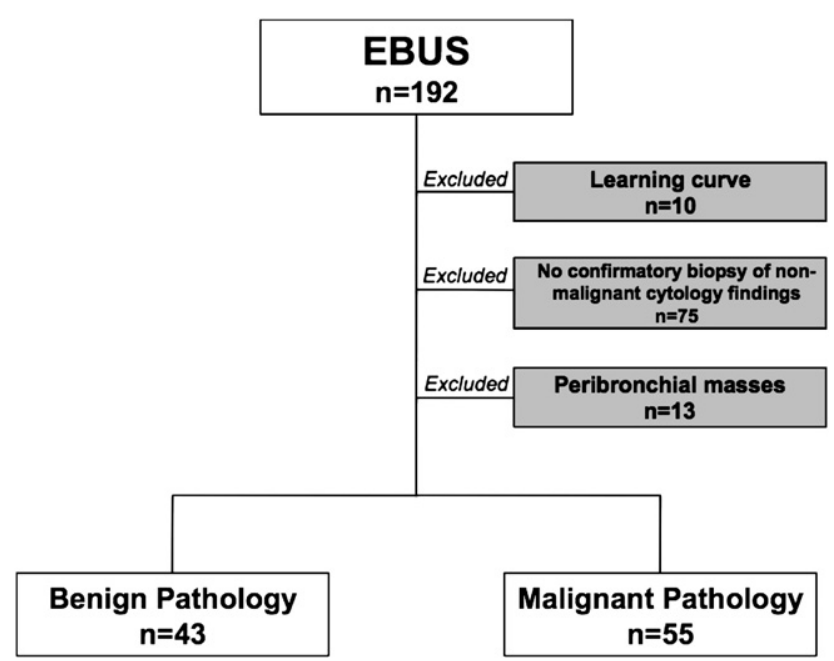

FIGURE 1. Patients included in and excluded from the study. EBUS, Endobronchial ultrasound.

principles when staging patients with NSCLC via EBUSFNA, and (3) perform additional procedures at the same time as EBUS-FNA to streamline patient care.

\section{Accurate Use of Endobronchial Ultrasound-Guided Fine-Needle Aspiration}

At the University of Minnesota, EBUS-FNA had an overall sensitivity of $88.9 \%$, a specificity of $96 \%$, an PPV of $98.5 \%$, an NPV of $75 \%$, and an accuracy of $90.7 \%$. Our series is unique, because $43.9 \%$ of our patients had a benign final pathologic diagnosis, and these performance measures

TABLE 1. Final pathologic diagnoses

\begin{tabular}{lcc}
\hline & No. & Percent \\
\cline { 2 - 3 } \multicolumn{1}{c}{ Diagnosis } & $\mathbf{n = 9 8}$ & $\%$ \\
\hline Benign pathology & 25 & \\
Normal & 15 & 25.5 \\
Granuloma & 3 & 15.3 \\
Hyperplasia & & 3.1 \\
Primary lung cancer & 33 & \\
NSCLC & 5 & 33.7 \\
SCLC & & 5.1 \\
Other malignancies & 4 & \\
Esophageal & 3 & 4.1 \\
Lymphoma & 2 & 3.1 \\
Breast & 1 & 2.0 \\
Uterine & 1 & 1.0 \\
Transitional cell & 1 & 1.0 \\
Melanoma & 1 & 1.0 \\
Colorectal & 1 & 1.0 \\
Duodenal & 1 & 1.0 \\
Renal cell & 1 & 1.0 \\
Thyroid & 1 & 1.0 \\
Mesothelioma & & 1.0 \\
\hline$N S C L$ Non-small cell lung cancer; $S C L C$, small cell lung cancer.
\end{tabular}

NSCLC, Non-small cell lung cancer; $S C L C$, small cell lung cancer.
TABLE 2. Summary of cases of rapid on-site evaluation and permanent cytology discrepancy

\begin{tabular}{ll}
\hline \multicolumn{1}{c}{ ROSE results } & Final cytology \\
\hline Nondiagnostic & NSCLC \\
Necrotic lymph node & NSCLC \\
Nondiagnostic & Granuloma \\
Benign lymph node & Sarcoid \\
Necrotic lymph node & NSCLC \\
Total: & $5(5.2 \%)$ \\
\hline$N S C L C$, Non-small cell lung cancer; $R O S E$, rapid on-site evaluation.
\end{tabular}

reflect the use of EBUS-FNA as a diagnostic tool for indications other than lung cancer. No data published to date have used surgical biopsy to verify the performance of EBUSFNA in benign disease. ${ }^{17,18}$ For benign disease, the sensitivity was lower than the sensitivity for malignant disease $(72.2 \%$ vs $87.9 \%)$. Nonetheless, we now first evaluate all patients with suspected benign mediastinal lymphadenopathy with EBUS-FNA; if ROSE demonstrates benign pathology (ie, granuloma, hyperplasia) and the clinical suspicion for a malignant process is low, we do not perform any other confirmatory procedure. However, if the sample is negative or nondiagnostic, we will obtain a surgical biopsy in the same anesthesia setting.

EBUS-FNA in patients with cancer had a sensitivity of $87.9 \%$, an NPV of $84.4 \%$, and an accuracy of $91.7 \%$. These values compare favorably with the published values for EBUS-FNA in lung cancer. ${ }^{1-7}$ In addition, our values also compare well with the results of mediastinoscopy in patients with cancer. ${ }^{8-14,19}$ The NPV of EBUS-FNA in cancer is still somewhat lower than that of mediastinoscopy. In patients with suspected malignancy, a negative EBUS-FNA sample must be interpreted in the context of the pretest probability of cancer in the sampled MLN. We strongly encourage verification of a negative ROSE result if the clinician has any suspicion that the MLN is potentially positive for malignancy.

We observed $1 \mathrm{FP}$ result in a patient with squamous cell cancer of the left distal mainstem bronchus. This patient underwent an EBUS-FNA of MLN station 7 through the right mainstem bronchus in an attempt to minimize the possibility of contamination with dysplastic bronchial cells; the cytology result was positive. Unique clinical circumstances led the patient to undergo a left pneumonectomy. At the time

TABLE 3. Summary of predictive measures for endobronchial ultrasound-guided fine-needle aspiration based on immediate pathology

\begin{tabular}{lccc}
\hline & Overall & Cancer diagnosis & Benign diagnosis \\
\hline Sensitivity (\%) & 88.9 & 87.9 & 72.2 \\
Specificity (\%) & 96.0 & 97.4 & 95.8 \\
PPV (\%) & 98.5 & 98.1 & 92.8 \\
NPV (\%) & 75.0 & 84.4 & 82.1 \\
Accuracy (\%) & 90.7 & 91.7 & 85.7 \\
\hline
\end{tabular}

$P P V$, Positive predictive value; $N P V$, negative predictive value. 
TABLE 4. Summary of additional procedures performed at the time of endobronchial ultrasound-guided fine-needle aspiration

\begin{tabular}{lcc}
\hline \multicolumn{1}{c}{ Procedure } & No. performed & $\%$ \\
\hline Diagnostic & & \\
Mediastinoscopy & 25 & 26.3 \\
EUS & 15 & 15.8 \\
MLND (open or & 14 & 14.3 \\
$\quad$ thoracoscopic) & & \\
Therapeutic & 13 & 13.7 \\
Lobectomy & 8 & 8.4 \\
Pulmonary wedge resection & & \\
Other & 4 & 4.2 \\
EGD & 4 & 4.2 \\
PEG & 4 & 1.0 \\
Port-a-catheter placement & 1 & \\
Pleurodesis & 88 & \\
Total additional procedures: & & \\
\hline
\end{tabular}

EUS, Endoscopic ultrasound (esophageal); $M L N D$, mediastinal lymph node dissection; $E G D$, esophagogastroduodenoscopy; $P E G$, percutaneous endoscopic gastrostomy.

of surgery, carcinoma in situ was evident in the proximal right mainstem bronchus requiring a carinal resection. Surgical pathology of the subcarinal lymph nodes was negative. We concluded that the presence of carcinoma in situ at the proximal right mainstem bronchus contaminated our EBSU-FNA specimen, leading to an FP result. Consequently, we now sample MLN station 7 via esophageal endoscopic ultrasound (EUS) in patients with central squamous cell cancers or if any suspicion exists that an FP result is a possibility. To our knowledge, this is the first report of an FP EBUS-FNA result in the literature.

\section{Appropriate Oncologic Staging With Endobronchial Ultrasound-Guided Fine-Needle Aspiration}

At least 3 N2 MLN stations (4R, 4L, and 7) should be sampled when staging potentially operable NSCLC. To our knowledge, this is the first report in the literature indicating that 3 MLN stations per patient can be routinely sampled with EBUS-FNA (previous publications sampled only an average of 1.5 MLN stations per patient), indicating that EBUS-FNA can be used as a staging tool while adhering to oncologic principles. ${ }^{7,17} \mathrm{We}$ also emphasize that relying on ultrasonographic criteria alone to determine whether an MLN is benign or malignant is fraught with errors, as evidenced by the experience with EUS. ${ }^{20}$ FNA is recommended even for benign-appearing MLNs.

\section{Effective Patient Care}

Thoracic surgeons who perform EBUS-FNA have the unique opportunity to streamline patient care because of their ability to perform any necessary additional diagnostic or therapeutic procedure in the same anesthesia setting. We performed at least 1 additional procedure in $50 \%$ of our patients at the same time as EBUS-FNA. Confirmatory surgical biopsies (eg, mediastinoscopy, thoracoscopy) are performed immediately, if necessary. In addition, we will perform EUS in the same setting, commonly with the EBUS bronchoscope, under the following circumstances: (1) failed sampling or a nondiagnostic sample of MLN stations $2 \mathrm{R}, 2 \mathrm{~L}, 4 \mathrm{~L}$, or 7; (2) concern of a potential FP sample in patients with diffuse airway metaplasia or dysplasia; or (3) sampling of MLN stations 8 and 9, left adrenal gland, or liver lesions if indicated.

We often perform EBUS-FNA as a staging procedure in the same setting as a pulmonary resection. On the basis of our last 31 procedures, an average EBUS-FNA takes 46.5 minutes from start to official ROSE reading by the cytologist; this is not an unreasonable amount of time if combined with a pulmonary resection. Of note, the procedural time of a mediastinoscopy, from the start of positioning to skin closure, is shorter than that for EBUS-FNA; however, the time to process a frozen section is longer than the time required for ROSE. Preliminary observations at our the University of Minnesota suggest that an expeditious mediastinoscopy saves approximately 10 minutes when compared with EBUSFNA (unpublished data, Rafael Andrade, MD, 2009). Finally, a thoracic surgeon can perform other therapeutic or palliative interventions (eg, intravenous port placement, gastrostomy tube placement, pleurodesis) in the same setting as EBUS-FNA.

\section{Limitations}

Our study is retrospective and included only patients with malignant EBUS-FNA findings or benign disease confirmed by a surgical biopsy; we acknowledge the potential for selection bias given our study design. In addition, the focused experience of a single operator and a small group of expert pathologists who are always available for ROSE will not universally be reproducible in clinical practice. The best way to determine the diagnostic performance of EBUS-FNA will be to carry out a prospective, multi-institutional study that will directly compare EBUS-FNA with surgical biopsy.

\section{Recommendations}

We suggest that thoracic surgeons who want to start a successful EBUS-FNA program work in close relationship with pathologists. Our recommendations to minimize diagnostic inaccuracies are as follows:

1. Patients with suspected or confirmed malignant disease: An FN result in a patient with malignant disease can have serious clinical repercussions; for this reason, a novice EBUS-FNA team should initially confirm every nonmalignant result with a surgical biopsy. Experienced EBUSFNA teams should corroborate nonmalignant cytology results in accordance with the clinical situation.

2. Patients with suspected benign disease: The evaluation of patients with clinically suspected benign disease 
depends on the differential diagnoses and the EBUSFNA team's experience. We recommend initial verification of every benign or nondiagnostic cytology finding with a surgical biopsy, and then the EBUS-FNA team will have accurately determined the diagnostic performance of EBUS-FNA for benign disease on its own.

\section{CONCLUSIONS}

We conclude that it is imperative for thoracic surgeons to become facile with EBUS-FNA for several reasons: (1) to accurately use EBUS-FNA as a diagnostic tool in patients with thoracic diseases, (2) to perform EBUS-FNA following appropriate oncologic principles (ie, proper sampling of standard MLN stations); and (3) to streamline patient care, because thoracic surgeons have the ability to perform additional interventions at the same time as EBUS-FNA.

\section{References}

1. Gu P, Zhao YZ, Jiang LY, Zhang W, Xin Y, Han BH. Endobronchial ultrasoundguided transbronchial needle aspiration for staging of lung cancer: a systematic review and meta-analysis. Eur J Cancer. 2009;45:1389-96.

2. Groth SS, Whitson BA, D'Cunha J, Maddaus MA, Alsharif M, Andrade RS. Endobronchial ultrasound-guided fine-needle aspiration of mediastinal lymph nodes: a single institution's early learning curve. Ann Thorac Surg. 2008;86: 1104-10.

3. Herth FJ, Becker HD, Ernst A. Ultrasound-guided transbronchial needle aspiration: an experience in 242 patients. Chest. 2003;123:604-7.

4. Vincent BD, El-Bayoumi E, Hoffman B, Doelken P, DeRosimo J, Reed C, et al. Real-time endobronchial ultrasound-guided transbronchial lymph node aspiration. Ann Thorac Surg. 2008;85:224-30.

5. Yasufuku K, Chiyo M, Sekine Y, Chhajed PN, Shibuya K, Iizasa T, et al. Realtime endobronchial ultrasound-guided transbronchial needle aspiration of mediastinal and hilar lymph nodes. Chest. 2004;126:122-8.

6. Rintoul RC, Tournoy KG, El Daly H, Carroll NR, Buttery RC, van Kralingen K, et al. EBUS-TBNA for the clarification of PET positive intra-thoracic lymph nodes-an international multi-centre experience. J Thorac Oncol. 2009;4:44-8.

7. Szlubowski A, Kuzdzal J, Kolodziej M, Soja J, Pankowski J, Obrochta A, et al. Endobronchial ultrasound-guided needle aspiration in the non-small cell lung cancer staging. Eur J Cardiothorac Surg. 2009;35:332-6.

8. Coughlin M, Deslauriers J, Beaulieu M, Fournier B, Piraux M, Rouleau J, et al. Role of mediastinoscopy in pretreatment staging of patients with primary lung cancer. Ann Thorac Surg. 1985;40:556-60.

9. Cybulsky IJ, Bennett WF. Mediastinoscopy as a routine outpatient procedure. Ann Thorac Surg. 1994;58:176-8.

10. Hammoud ZT, Anderson RC, Meyers BF, Guthrie TJ, Roper CL, Cooper JD, et al. The current role of mediastinoscopy in the evaluation of thoracic disease. JThorac Cardiovasc Surg. 1999;118:894-9.

11. Lemaire A, Nikolic I, Petersen T, Haney JC, Toloza EM, Harpole DH Jr, et al. Nine-year single center experience with cervical mediastinoscopy: complications and false negative rate. Ann Thorac Surg. 2006;82:1185-90.

12. Luke WP, Pearson FG, Todd TR, Patterson GA, Cooper JD. Prospective evaluation of mediastinoscopy for assessment of carcinoma of the lung. J Thorac Cardiovasc Surg. 1986;91:53-6.

13. Patterson GA, Ginsberg RJ, Poon PY, Cooper JD, Goldberg M, Jones D, et al. A prospective evaluation of magnetic resonance imaging, computed tomography, and mediastinoscopy in the preoperative assessment of mediastinal node status in bronchogenic carcinoma. J Thorac Cardiovasc Surg. 1987;94:679-84.

14. Pauwels M, Van Schil P, De Backer W, Van den Brande F, Eyskens E. Repeat mediastinoscopy in the staging of lung cancer. Eur J Cardiothorac Surg. 1998; 14:271-3.

15. Groth SS, Andrade RS. Endobronchial ultrasound-guided transbronchial needle aspiration for mediastinal lymph node staging in non-small cell lung cancer. Semin Thorac Cardiovasc Surg. 2008;20:274-8.
16. Herth FJ, Eberhardt R, Vilmann P, Krasnik M, Ernst A. Real-time endobronchial ultrasound guided transbronchial needle aspiration for sampling mediastinal lymph nodes. Thorax. 2006;61:795-8.

17. Herth FJ, Eberhardt R, Krasnik M, Ernst A. Endobronchial ultrasound-guided transbronchial needle aspiration of lymph nodes in the radiologically and positron emission tomography-normal mediastinum in patients with lung cancer. Chest. 2008; 133:887-91.

18. Alsharif M, Andrade RS, Groth SS, Stelow EB, Pambuccian SE. Endobronchial ultrasound-guided transbronchial fine-needle aspiration: the University of Minnesota experience, with emphasis on usefulness, adequacy assessment, and diagnostic difficulties. Am J Clin Pathol. 2008;130:434-43.

19. Baker JJ, Solanki PH, Schenk DA, Van Pelt C, Ramzy I. Transbronchial fine needle aspiration of the mediastinum. Importance of lymphocytes as an indicator of specimen adequacy. Acta Cytol. 1990;34:517-23.

20. Puli SR, Reddy JBK, Bechtold ML, Antillon D, Ibdah JA, Antillon MR. Staging accuracy of esophageal cancer by endoscopic ultrasound: a meta-analysis and systematic review. World J Gastroenterol. 2008;14:1479-90.

\section{Discussion}

Dr Hiran Fernando (Boston, Mass). Dr Andrade, a common clinical challenge for thoracic surgeons is accurately diagnosing a patient with a specific benign pathology, such as sarcoidosis, and often these patients will have a number of diagnostic procedures before referral to a thoracic surgeon, including transbronchial needle aspiration or fine-needle aspiration. You describe success rates for benign and malignant diagnoses, and you separate these in Table 3 in your article. You mention that you have a lower sensitivity for a benign diagnosis of $72.7 \%$ versus $87.9 \%$ for malignancy. Do you mean that this $72.7 \%$ was simply to make a benign rather than a cancer diagnosis or does this sensitivity refer to actually making a specific benign diagnosis, such as sarcoid or histoplasmosis? If you suspect that a patient up front has a benign diagnosis in a lymph node station that would be accessible by mediastinoscopy where you would get the whole lymph node, would you recommend mediastinoscopy for that patient or an initial EBUS on the basis of your results, and what would be your reasoning for your approach?

Dr Andrade. In terms of the first question, the way we broke the benign analysis down was by presumptive diagnosis. That's what the calculation reflects. Can you repeat your second question once again, please?

Dr Fernando. If you have a specific benign cause, for instance, if a pulmonologist sends you a patient with a possible diagnosis of sarcoidosis, would you recommend EBUS as an initial approach or would you go straight to mediastinoscopy where you can get lymph node architecture?

Dr Andrade. A lot of it depends on your pretest probability. The main thing you want to avoid is missing a malignancy. If a patient has a clinical picture suggestive of sarcoid, and EBUS-FNA cytology demonstrates sarcoid that is noncaseating granuloma, I will stop there. However, all of my patients provide consent for mediastinoscopy, and I tell them if there's any doubt, we will proceed with a mediastinoscopy.

Dr Fernando. So your initial approach would still be EBUS for those patients?

Dr Andrade. Yes.

Dr Fernando. Another question going on from that is, when you have a patient who has a known diagnosis of cancer but who does not have MLN enlargement by computed tomography or positron emission tomography abnormalities, do you recommend EBUS in those patients with clinically normal lymph nodes on the basis 
of their imaging or would you perform mediastinoscopy in those patients?

Dr Andrade. I think that decision depends on the surgeon. In my practice, a patient with a normal mediastinum and early-stage cancer will undergo an EBUS. If I obtain good samples on ROSE intraoperatively, I will then proceed with a lobectomy. However, I would not encourage surgeons who are not experienced with EBUS to do this until they have passed their learning curve.

Dr Fernando. You still have a fairly significant NPV for mediastinoscopy, so why perform the EBUS if you're going to then perform a thoracoscopy? Are you going to then go on to perform a mediastinoscopy after the EBUS?

Dr Andrade. There are 2 points here. One, are you going to stage the mediastinum or not. That's another point of discussion. By assuming that one decides to stage the mediastinum, and on the basis of our experience with nearly 200 EBUS procedures, I can now confidently say that if the clinical stage is I and the ROSE demonstrates a good sample with benign lymphocytes, I do not confirm it with mediastinoscopy. However, if I have any shred of doubt, I will do a mediastinoscopy.

Dr Thomas D'Amico (Durham, NC). That was a good study, well designed and well performed. I have 2 questions. Did you say if you had any complications from your 200 EBUS-FNAs? Were there any significant complications?

Dr Andrade. No, I didn't say. If you look at all of them, we had 3 patients out of almost 200 who stayed overnight for respiratory decompensation. That has been the only complication we have had so far.

Dr D'Amico. I think the 2 most important findings, other than how well your NPV is, are that you have twice as many lymph nodes per station, as in much of the literature, for which you are to be congratulated, and that you trust ROSE, which a lot of other practitioners have given up on. How have you perfected ROSE to the point that you can trust it to do a secondary procedure?

Dr Andrade. We are fortunate to have outstanding pathologists at our institution. I think that is the only reason we can get away with that. They have extensive experience with transbronchial needle aspiration biopsy. A cytologist has to have specific experience in this to be able to interpret these samples reliably.

Dr F. Detterbeck (New Haven, CT). First, I think it's more important to calculate things by patient and not by node. You artifi- cially make your numbers look better when you calculate it by node, and I think you really have to do it by patient. Second, I think you really can't make a statement about the usefulness of this for staging lung cancer when you're also mixing in people and just trying to get a diagnosis of mediastinal tissue, such as for sarcoid. I think you have to separate that. Third, this is an important contribution. I think it is an important fact that you have done this carefully. It makes a difference whether somebody is invested in a technique and does it well. You have sampled a lot of nodes and you have been careful to look at your results, and I can tell you that I think EBUS looks good in some literature reports, but in some places where it's done, where people stick 1 node and then get out and don't do a good job, they are not going to get nearly as good results. Thoracic surgeons who have a vested interest in appropriately staging the mediastinum are more likely to do a good job, such as you have done.

Dr Andrade. Thank you.

Dr Shaf Keshavjee (Toronto, Ontario, Canada). In that one case with an FP, is it your practice to change your needle for each lymph node station? Also, did you look in retrospect at what the cause was of that FP reading? Were the cytopathologists reading the contamination of cells from the airway on the way in?

Dr Andrade. The first answer is yes, we do change our needle. If we first diagnose a malignant lymph node, we do not use that needle again. In response to the FP case, this particular patient had a distal left main stem squamous cell cancer. I purposefully biopsied the subcarinal lymph nodes through the right main stem bronchus to avoid any potential contamination. Eventually, he underwent surgery and required a carinal pneumonectomy because there was a carcinoma in situ extending all the way across the carina into the proximal right main stem. What I do now if I have any suspicion of contaminations is to biopsy level 7 via EUS or mediastinoscopy.

Dr Nasser Altorki (New York, NY). Rafael, I have a quick question for you. Do you have any experience with diagnosis of N1 stations?

Dr Andrade. Yes. We didn't mention it here. We do sample N1 lymph nodes. We don't do it as a staging procedure. We do it more in patients with a diagnosis of probable lung cancer and we're sampling to get a tissue diagnosis. 